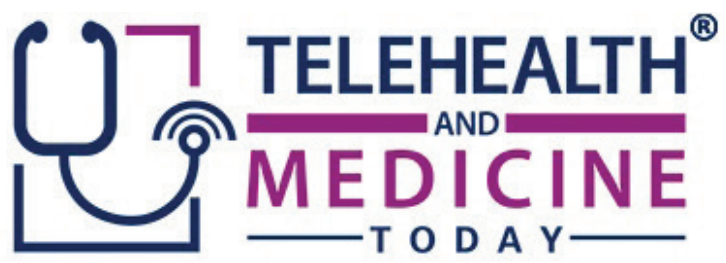

\title{
Enhancing Digital Empathy and Reimagining the Telehealth Experience
}

\author{
Matthew Sakumoto, $\mathrm{MD}^{1} \odot$ and Sarah $\operatorname{Krug}^{2,3}$
}

Affiliations: ${ }^{1}$ Sutter Health, San Francisco, CA, USA; ${ }^{2}$ The Health Collaboratory, New York, NY, USA;

${ }^{3}$ CANCER101, New York, NY, USA

Corresponding Author: Matthew Sakumoto, Email: matthew.sakumoto@ucsf.edu

Keywords: Patient Perspective, Digital Empathy, Telehealth Literacy, Patient Centricity

Section: Opinion/Perspective/Point of View

As telehealth is increasingly adopted across all care settings, it is important to understand how clinicians can adapt and respond to patient needs. Drawing from the experiences of a virtual primary care physician and a patient advocate, this Perspectives editorial provides additional insights beyond the telehealth basics for establishing digital empathy and a remote therapeutic connection.

$\mathrm{D}$ uring the coronavirus disease 2019 (COVID-19) pandemic, the seismic shift to telehealth was crucial to patients and health systems to provide care at a distance. While telehealth has existed since the 1970s to support rural health and is utilized by integrated health systems like Kaiser and the Veterans Affairs, in 2020, thousands of new patients and clinicians experienced telehealth for the first time. With this surge, we also felt the growing pains of adopting and adapting to this new virtual care medium. ${ }^{1}$ Some best practices have been developed, such as webcam positioning, how to maintain good eye contact, and making sure both the patient and clinician are in safe, quiet, and private spaces. ${ }^{2}$ However, in this Perspectives piece, we hope to move beyond the Telehealth 1.0 basics and delve into more advanced concepts to elevate the telehealth experience. The author's organization, The Health Collaboratory, ${ }^{3}$ led a series of co-design sessions with patients and their care partners to understand barriers they encountered, with the intent of creating guidance and education to prepare for all aspects of virtual care, addressing elements before, during, and after the visits. After reviewing both the existing peerreviewed literature ${ }^{2,4}$ and lay press articles, 5,6 we summarize our findings in Table 1 . We believe that clinicians and patients can co-design 
Table 1. Key elements identified to enhance the telehealth experience

How do we humanize the visit through digital empathy?

- Pay attention to video framing to include arms and torso, incorporating hand gestures and posture into non-verbal communication.

- Observe digital body language and non-verbal cues, and utilize the power of pause in natural conversation exchange to engage the patient during the virtual visit.

- Practice active listening, allowing the patient to narrate their story while encouraging dialog, and use the teach-back method to confirm that both the patient and clinician share the same understanding.

- Remember that time makes up for touch. In the absence of a traditional physical examination, taking the time to listen to the patient's history enhances the therapeutic relationship and the diagnostic process.

How do we empower patients, care partners, and clinicians with telehealth literacy?

- Invest in pre-visit preparation for both patients and clinicians. This may include a test visit for navigating the hardware and software interface, as well as upfront agenda-setting.

- Address privacy concerns with patients and offer suggestions for safer spaces.

- Provide educational opportunities for clinicians to learn unique telehealth skills such as webside manner, health literacy skills, and advanced, clinician-directed, patient-performed virtual exam maneuvers.

- Recognize that health inequities may be exacerbated without an assessment of a patient's needs, readiness, and proper support provided in the context of his or her life.

How do we reimagine the continuum of care through interstitial care and alignment of personal preferences?

- Use a virtual communication technology to expand the care team across time and distance by incorporating real-time video interpreters, care partners, family members, or clinical consultants.

- Increase patient engagement by aligning care delivery with patient preferences, and integrating patient-generated health data into chronic or complex disease management.

- Deploy therapeutics from the digital medicine cabinet to foster better mental health, preventative care, and wellness initiatives.

- Personalize the experience based on the needs of the patient and care partner, empowering them with the skills to decide on when a virtual visit or in-person visit may be appropriate.

- Co-design new telehealth quality measures that assess the experience of patients and clinicians.

the next evolution of telehealth by focusing on humanizing the visit by enhancing this connection through building confidence and competence in telehealth literacy, and together unlock the potential for novel models across the care continuum.

\section{HUMANIZING THE VISIT AND ENHANCING THE CONNECTION}

First impressions matter. In just seconds, a person decides whether someone is trustworthy, competent, or likeable. The research study shows that first impressions are most influenced by 
non-verbal communication, facial cues, and body language that can be harder to express virtually; therefore, attaining a strong virtual first impression may require more effort than an in-person interaction. ${ }^{7}$ These first impressions can be long lasting, and the initial telehealth encounter can influence a patient's receptivity to future virtual visits. Given the importance of the digital first impression, we identified digital empathy, nonverbal cues, active listening, and narrative-based medicine as key components to success.

The practice of empathy is a cornerstone in establishing a strong connection and emotional bridge between a patient and their clinician. Empathy is the ability to perceive another person's emotions, imagine their thoughts and feelings, and then have the skill to react appropriately. The importance of empathy in patient care and building trust over time is well documented, and is the foundation of a patientclinician therapeutic relationship. ${ }^{8}$ Ultimately, harnessing these skills can improve patient satisfaction and health outcomes. With the rapid adoption of social media and use of other technologies, our communication has changed, and digital interactions may occur without the empathetic social antenna and emotional cues that are experienced more naturally in an in-person exchange. Studies of healthcare communication have shown that the socioemotional tone of virtual communication could be improved by increasing patientcenteredness. ${ }^{9}$ The digitalization of healthcare has led to a boundless opportunity to connect meaningfully and share data beyond the constraints of time and geography. With these modern advances, it is important for us to recognize how the expression of empathy may also need to evolve through an understanding of the emerging science of "digital empathy." During a time when many patients may be adjusting to daily life stress exacerbated by the pandemic, they are also craving for compassion and want to feel understood.

Non-verbal cues and body language speak louder than words. A variety of non-verbal cues impart a richness of communication with in-person interactions, which is often lost in translation through virtual talking heads on a screen. In a study at a Veterans Health Administration clinic, this disconnection made patients sometimes "feel that they were not part of the conversation" during telehealth visits. ${ }^{10}$ To better convey and perceive non-verbal cues, we recommend widening the frame of view. As trivial as a frame might sound, the webcam is the window to the virtual visit. To utilize body language effectively, the position of the camera should maximize visualization of the torso and arms and avoid the "talking head" effect that a closely positioned webcam will cause. Repositioning allows for effective communication tools such as hand gestures and posture; posture, in particular, can affect a patient's perception of clinician engagement. To convey empathy and inclusiveness, clinicians can utilize an open stance. Tone, volume, and cadence also can influence a patient's engagement, and thus, it is important that the tone rises and falls naturally. Inflection can also help place importance on certain points.

Video visits introduce additional wrinkles to attend to during the visit. While eye contact through the webcam has been identified as an important element of "webside" manner, the clinician may miss important reactions, nonverbal cues, facial expressions, and body gestures that the patient makes. Practicing situational awareness requires periodic cycling between webcam eye contact, patient video feed, the clinician screen to ensure proper framing, and the medical record. These are just a few of the clinician telehealth literacy skills we discuss in the following section. 
An extension of empathy is active listening, where one takes the time to absorb, understand, and respond to what a patient is saying with unconditional acceptance and unbiased reflection, yet the virtual visit can pose new barriers to active listening. The patient should feel heard through body language or acknowledgment cues that do not interrupt the patient's story, such as a nod. Documenting the story in parallel to listening to it has its drawbacks where the sound of a clicking keyboard can make the patient feel like the clinician is not taking the time to listen. Another technology-induced barrier is the periodic audio delay that can disrupt the fast-paced exchange of conversation. This disrupts the natural flow of conversation, and can also lead to talking over each other. Slowing down, taking pauses, and using tone, volume, cadence, and enunciation carefully can influence perceptions, understanding, attitudes, and, ultimately, behavior. In a face-to-face interaction, how we speak is important; however, in a virtual visit, the delivery carries greater weight to compensate for key elements of the clinical visit, such as a lack of human touch. During in-person visits, clinicians listen to their patients for between 18 and 23 seconds before interrupting. ${ }^{11}$ Will audio delays exacerbate these interruptions? There is power in pause where slowing down speech during conversation can help ensure a patient understands important information and has time to process and reflect so that they can ask follow-up questions. Encouraging the patient to continue telling their story and helping them piece together clinically important elements can be done through occasional questions, comments, and a recap that shows you understood what they communicated. This "teach-back" method is a validated technique that has shown improved outcomes in diseasespecific knowledge, adherence, and self-efficacy. ${ }^{12,13}$
Finally, active listening is a key tenet of narrative-based medicine, which is a commitment to understanding the life and unique narrative of both the patient and their care partner. The patient's story and narrative of his or her illness is one of medicine's oldest diagnostic tools; however, it is often replaced by the clinician's story of disease that leans on evidence-based medicine and technology. The virtual visit also removes many aspects of the traditional physical examination, further increasing reliance on the patient's history and story. Building trust and empathy, and creating a safe space for the patient to share openly, is paramount to providing quality clinical care.

A diagnosis is only the beginning of a patient's story; his or her illness will continue to unfold and evolve over time. The patient's narrative not only illuminates the history of the condition but also allows us to contextualize and better understand their experience of the disease and the social challenges they may experience in getting better. Ultimately, this practice can prompt a shift from the traditional practice of "how do I treat this disease?" to "how do I help this person?" Virtual visits provide an important opportunity to practice the art of listening and truly embrace the patient's story.

\section{DEVELOPING TELEHEALTH LITERACY}

While making a human connection is most important, to foster these higher order connections, both patients and clinicians need to develop foundational "telehealth literacy." Developing this confidence and competence in telehealth communication skills takes time, and healthcare delivery organizations should invest time in the pre-visit preparation.

For patients, telehealth literacy means ensuring instructions are provided at an appropriate level of health literacy and technical literacy, and 
empowering and educating care team members to assist patients at all levels. Health literacy impacts a person's ability to obtain, process, and understand basic health information services to make "appropriate" health decisions, all of which has an impact on patient engagement and health outcomes. Health literacy now emphasizes how people can use health information, rather than just understand it, with a focus on supporting their ability to make "well-informed" decisions rather than merely appropriate ones. ${ }^{14}$ The Centers for Disease Control and Prevention (CDC) currently provides guidance and tools for health literacy such as the use of plain language with limited jargon, up-front agenda setting, encouraging questions, and avoiding information overload by emphasizing up to three key points, while also incorporating the teach-back method where patients explain key information in their own words to ensure that they understand the next steps. ${ }^{14}$ However, currently, there are no guidelines specific for telehealth. Other telehealth readiness tools examine patient engagement and health literacy, but more granular guidance is needed to identify gaps. ${ }^{15}$ With the advent of telehealth, we need to integrate the basic principles of health literacy, and empower patients and their families with the technical skills needed to use hardware and software to connect and communicate with the care team. The social dynamics of telehealth interactions will need more research studies to determine best practices to ensure it does not exacerbate health inequities.

Additional barriers to patient telehealth literacy are privacy concerns. While the four walls of the traditional office offer a level of security, patients have privacy concerns when communicating over video or messaging. While telehealth from the comfort of their home is convenient, patients may not always have the luxury of an extra bedroom or a private space to conduct the visit.
In the author's experience, taking the time to explain why clinical video software requires high levels of security, offering suggestions for higher privacy spaces such as the car or the bathroom, and converting the video visit to a phone call or in-person visit can sometimes alleviate these privacy concerns.

Developing clinician telehealth literacy is equally important. Clinicians need opportunities to hone these skills, and while the Association of American Medical Colleges has proposed telehealth competencies, ${ }^{16}$ there is no consistent exposure, feedback, or curriculum for trainees or those already practicing medicine. ${ }^{17}$ To practice telehealth at the top of their license, clinicians need to develop additional skill sets such as webside manner and alternative exam maneuvers for the virtual physical examination.

\section{REIMAGINING THE CONTINUUM OF CARE}

Humanizing the virtual visit and developing telehealth literacy have the potential to transform telehealth into simply health, normalizing the adoption of virtual care into everyday practice and introducing new models of care beyond the episodic clinic visits. For patients, telehealth has the potential to unlock interstitial care through improved access and care coordination. These new care opportunities connect the dots between the in-person visits and can engage the extended care team, including care partners and family members, interpreters, and clinical consultants (like endocrinology or oncology), along the patient's journey. The realm of patient-generated health data, whether collected through patient self-reported outcomes or though remote monitoring devices, fitness trackers, and other wearable devices can unlock insights into chronic disease management. The new digital medicine cabinet with behavior modification apps and online support programs can help our health 
system evolve past sick care and into preventative care and wellness promotion. Studies have shown that co-creation of communication tools have the potential to increase patient empowerment, and adoption and effective use of these new tools start with effective and empathetic patient-clinician communication. $^{18}$

From the clinician's perspective, aligning virtual care modalities with patient's preferences and the overall risks, costs, and benefits will be an important balancing act in this new care paradigm. For example, in the author's virtual-first primary care practice, we ask patients about their care team expectations (convenience, understanding/personal, or guidance/coaching). ${ }^{19}$ Their response informs our choice of outreach channels, and how we engage with our patients during and in between visits. In our ever-changing healthcare landscape, transparency is the key to maintaining this trust and communication.

Finally, the new continuum of virtual care holds great potential for addressing the quadruple aim (enhance patient experience, improve population health, reduce costs, and improve the work-life of healthcare providers). The American Medical Association's Return on Health framework has identified six distinct value streams of the digitally enabled hybrid care model. Beyond just financial performance, optimized virtual care can improve clinical outcomes, quality and safety, access to care, patient and family experience, and the clinician experience. As we explore the exciting new possibility of telehealth, it is important to continually assess for telehealth safety and effectiveness.

Areas of future study include looking at the effect of patient and clinician satisfaction and clinical outcomes, and if these enhanced communication skills can mitigate concerns that virtual care is too impersonal. In addition, when defining measures of success, it is important to incorporate patient perspectives into this metric design.

\section{CONCLUSIONS}

Digital communication has become a firmly embedded component of the healthcare ecosystem, which provides boundless opportunities to reimagine the continuum of care based on the preferences and needs of the patient and their care partner. We hope that this has highlighted the importance of digital empathy and investing in patient, care partner, and clinician telehealth literacy to enhance the digital therapeutic connection, while continuously addressing the evolving needs of patients, care partners, and clinicians.

Funding Statement: No external funding was utilized for the preparation of this manuscript.

Conflicts of Interest: Matthew Sakumoto is an editorial board member for Telehealth and Medicine Today, and reports personal fees for work pertaining to Clearstep Health. All other authors report no relevant conflicts of interest.

Contributors: The corresponding author certifies that all listed authors meet the following four criteria:

Substantial contributions to the conception or design of the work; or the acquisition, analysis, or interpretation of data for the work; drafting the work or revising it critically for important intellectual content; AND final approval of the version to be published; AND

agreement to be accountable for all aspects of the work in ensuring that questions related to the accuracy or integrity of any part of the work are appropriately investigated and resolved. 


\section{REFERENCES}

1. Hollander JE, Carr BG. Virtually perfect? Telemedicine for Covid-19. N Engl J Med. 2020;382:1679-81. https://doi.org/10.1056/ NEJMp2003539

2. McConnochie KM. Webside manner: a key to high-quality primary care telemedicine for all. Telemed J E Health. 2019;25(11):100711. https://doi.org/10.1089/tmj.2018.0274

3. The Health Collaboratory. About. Available from: https://www.healthcollaboratory.com. [cited 3 October 2021].

4. Benziger CP, Huffman MD, Sweis RN, Stone NJ. The telehealth ten: a guide for a patient-assisted virtual physical examination. Am J Med. 2021;134(1):48-51. https://doi. org/10.1016/j.amjmed.2020.06.015

5. Joshi AU, Lewiss RE. Doctors offer advice for how to prepare for your telehealth visit. Dec. 31. Available from: https://www. inquirer.com/health/expert-opinions/doctortips-telehealth-maximize-visit-20201231. html [cited 2 October 2021].

6. Elias P. As telehealth visits become more common, here are ways you can make the most of your consultation. Washington Post. April 3. Available from https://www. washingtonpost.com/health/telehealthtips/2021/04/02/623c880c-712d-11eb85fa-e0ccb3660358_story.html [cited 2 October 2021].

7. Kimball AB, Morgan N. Building trust into telehealth. Harv Bus Rev. 24 March 2021. Available from: https://hbr.org/2021/03/ building-trust-into-telehealth [cited 2 October 2021].

8. Moudatsou M, Stavropoulou A, Philalithis A, Koukouli S. The role of empathy in health and social care professionals. Healthcare. 2020;8(1):26. https://doi. org/10.3390/healthcare8010026

9. Hogan TP, Luger TM, Volkman JE, Rocheleau M, Mueller N, Barker AM, et al. Patient centeredness in electronic communication: evaluation of patientto-health care team secure messaging. J Med Internet Res. 2018;20(3):e82. https://doi.org/10.2196/jmir.8801
10. Gordon HS, Solanki P, Bokhour BG, Gopal RK. "I'm not feeling like I'm part of the conversation' Patients" perspectives on communicating in clinical video telehealth visits. J Gen Intern Med. 2020;35(6):1751-58. https://doi.org/10.1007/s11606-020-05673-w

11. Phillips KA, Ospina NS. Physicians interrupting patients. JAMA 2017;318(1): 93-4. https://doi.org/10.1001/jama.2017.6493

12. Dinh TTH, Bonner A, Clark R, Ramsbotham J, Hines S. The effectiveness of the teach-back method on adherence and self-management in health education for people with chronic disease: a systematic review. JBI Database Syst Rev Implement Rep. 2016;14(1):210-47. https://doi. org/10.11124/jbisrir-2016-2296

13. Agency for Healthcare Research and Quality. Use the teach-back method: tool \#5. 2020. Available from: http://www.ahrq. gov/health-literacy/improve/precautions/ tool5.html [cited 3 October 2021].

14. CDC. Communication Guidance. CDC. gov; 2021. Available from: https://www. cdc.gov/healthliteracy/developmaterials/ guidancestandards.html [cited 29 September 2021].

15. Haque S. Telehealth readiness assessment tool. RTI Center for Health Care Advancement. Available from: https:// healthcare.rti.org/project/development-oftelehealth-readiness-assessment-tool [cited 29 September 2021].

16. Association of American Medical Colleges. Telehealth competencies. AAMC; 2021. Available from: https://www.aamc.org/ data-reports/report/telehealth-competencies [cited 14 April 2021].

17. Sakumoto M, Jelinek R, Joshi AU. Identification of gaps in graduate medical education telehealth training. Telehealth Med Today. Published online 30 July 2021;6. https://doi.org/10.30953/tmt.v6.276

18. Rezaei AA, Watson J, Cliff C, Miah SJ. Improving the theoretical understanding toward patient-driven health care innovation through online value cocreation: systematic review. J Med Internet 
Res. 2020;22(4):e16324. https://doi. org/10.2196/16324

19. Sutter Health. Tera practice: primary care that fits your life. 2021. Available from: https://www.sutterhealth.org/lp/terapractice/ index.html [cited 3 October 2021].

Copyright Ownership: This is an open access article distributed in accordance with the Creative Commons Attribution Non Commercial (CC BY-NC 4.0) license, which permits others to distribute, adapt, enhance this work non-commercially, and license their derivative works on different terms, provided the original work is properly cited and the use is noncommercial. See: http://creativecommons. org/licenses/by-nc/4.0. 